\title{
Efecto de la infección subclínica de Salmonella Typhimurium sobre los parámetros productivos en la producción de cuyes de engorde (Cavia porcellus)
}

\author{
Effect of subclinical infection of Salmonella Typhimurium on the productive \\ parameters in the production of fattening guinea pigs (Cavia porcellus)
}

Víctor Bazán R. ${ }^{1,3}$, Sandra Bezada Q.², Fernando Carcelén C. ${ }^{2}$, Graciela Yamada A. ${ }^{1}$

\section{RESUMEN}

Con el objetivo de determinar el efecto de la Salmonella Typhimurium sobre los parámetros productivos de cuyes (Cavia porcellus) se utilizaron 40 cuyes machos recién destetados, alimentados con una dieta base. Los cuyes fueron distribuidos en cuatro tratamientos con 10 repeticiones cada uno. T1: solución salina (control); T2: solución salina + APC (antibiótico promotor de crecimiento); T3: desafío con Salmonella Typhimurium; T4: desafío con Salmonella Typhimurium + APC. La dosificación con solución salina o con una dosis infectiva de Salmonella Typhimurium ( $2 \times 10^{6}$ UFC) se hizo en el día 11 del estudio. Se evaluó la ganancia de peso, consumo de alimento, conversión alimenticia, rendimiento de carcasa y la retribución económica. Se tomaron muestras al beneficio de órganos y de la carne para el estudio microbiológico. Los animales desafiados con Salmonella (T3 y T4) mostraron una menor ganancia de peso, mayor consumo de alimento y pobre índice de conversión alimenticia en comparación con los cuyes de T1 y T2 $(\mathrm{p}<0.05)$. El rendimiento de carcasa fue similar entre los cuatro tratamientos, mientras que la retribución económica en los cuyes de T3 y T4 fue afectada entre un 28 y $30 \%$. Se aisló Salmonella de dos muestras de pulmón y una de bazo en cuyes del T1, mientras que más del $50 \%$ de las muestras en cuyes de T3 y T4 se pudo aislar el patógeno. Solo se detectaron cuatro muestras con presencia de Salmonella en la carne (T3). Se concluye que los parámetros productivos y la retribución económica en cuyes se afectan negativamente por la infección subclínica con Salmonella Typhimurium.

Palabras clave: Salmonella Typhimurium; cuyes de engorde; parámetros productivos; ganancia de peso; conversión alimenticia

\footnotetext{
${ }^{1}$ Estación Experimental Huaral, Centro de Investigación IVITA, Facultad de Medicina Veterinaria, Universidad Nacional Mayor de San Marcos, Lima, Perú

${ }^{2}$ Laboratorio de Bioquímica, Nutrición y Alimentación Animal, Facultad de Medicina Veterinaria, Universidad Nacional Mayor de San Marcos, Lima, Perú

${ }^{3}$ E-mail:vbazanr@unmsm.edu.pe
}

Recibido: 19 de marzo de 2019

Aceptado para publicación: 30 de noviembre de 2019 
The effect of Salmonella Typhimurium was determined on the productive parameters of guinea pigs (Cavia porcellus). Forty recently weaned male guinea pigs fed with a base diet were used. The animals were distributed in four treatments with 10 repetitions each were used. T1: saline solution (control); T2: saline + AGP (antibiotic growth promoter antibiotic); T3: challenge with Salmonella Typhimurium; T4: challenge with Salmonella Typhimurium + AGP. Dosing with saline or with an infective dose of Salmonella Typhimurium $\left(2 \times 10^{6} \mathrm{CFU}\right)$ was made on day 11 of the study. Bodyweight gain, feed consumption, feed conversion, carcass yield and economic gain were evaluated. Samples were taken from organs and meat after the slaughtering for the microbiological analysis. Animals challenged with Salmonella (T3 and T4) showed lower bodyweight gain, higher feed intake and poorer feed conversion compared to guinea pigs of T1 and T2 $(\mathrm{p}<0.05)$. The carcass yield was similar in the four treatments, while the economic gain in guinea pigs of T3 and T4 was affected by $28-30 \%$. Salmonella was isolated from two lung samples and one spleen sample in animals from $\mathrm{T} 1$, while the pathogen could be isolated in more than $50 \%$ of the samples in animals of T3 and T4. Only four samples with Salmonella in the meat were detected (T3). It is concluded that the productive parameters and the economic retribution in guinea pigs are negatively affected by subclinical infection with Salmonella Typhimurium.

Key words: Salmonella Typhimurium; fattening guinea pigs; productive parameters; bodyweight gain; feed conversion

\section{INTRODUCCIÓN}

El cuy es un roedor herbívoro monogástrico, que presenta un estómago donde se inicia la digestión enzimática del alimento, pasa por el intestino delgado y llega al ciego donde realiza la fermentación bacteriana (Chauca, 1994). Esta característica le permite tener una alta eficacia en el momento de la digestión de la fibra y poder utilizar más eficientemente el alimento, de manera muy similar a lo presentado en caballos (Hirikawa, 2001).

El auge que viene presentando la crianza de cuyes (Cavia porcellus) ha propiciado un aumento en la presentación de enfermedades bacterianas, tales como la salmonelosis, que puede provocar hasta el $95 \%$ de morbilidad (Chauca, 1997) y alta mortalidad en crías durante la lactación (38-56\% en crianzas familiares, $23 \%$ en crianzas tecnificadas) (Ordoñez, 1998), considerándose como una de las enfermedades de mayor importancia en cuyes (Bustamante, 1993; Chauca et al., 1997; Molina, 2008). Los cuyes que se infectan y sobreviven terminan siendo portadores y diseminadores de esta enfermedad (Chero, 2015).

Existen especies de Salmonella que se desarrollan en el medio ambiente y otras que llegan a habitar en el agua y en el suelo (Carroll, 2014). El serotipo de mayor frecuencia en cuyes es la Salmonella enterica subespecie enterica serovar Typhimurium, llegando a presentarse en el $95 \%$ de los casos con relación a otros serotipos (Layme, 2010). La vía de transmisión más importante es la indirecta, ya sea por el consumo de alimentos o agua contaminada con heces de animales como roedores y aves infectados (Matsuura et al., 2010). La salmonelosis aguda en cuyes presenta un cuadro septicémico durante las primeras 24-48 horas de la infección, causando mortalidad, a veces sin pre- 
sentar signos, o con cuadros de inapetencia, decaimiento, pelo hirsuto y, en casos extremos, con parálisis de los miembros posteriores (Evans, 2005; Morales et al., 2007; Borrelli, 2011). Por otro lado, la infección puede permanecer por varios meses en casos de animales portadores asintomáticos.

El objetivo del presente estudio fue determinar el efecto de una infección asintomática de Salmonella Typhimurium sobre los parámetros productivos de cuyes y sobre el mérito económico de su crianza.

\section{Materiales y MéTOdos}

El estudio se realizó entre junio y setiembre de 2017 en la unidad de investigación de cuyes de la Facultad de Medicina Veterinaria (FMV) de la Universidad Nacional Mayor de San Marcos (UNMSM), en San Borja, Lima. Se utilizaron 40 cuyes mejorados machos destetados de la línea cárnica «G», destetados de dos semanas de edad, con peso promedio al destete de $241 \mathrm{~g}$. Los cuyes provinieron del módulo demostrativo de cuyes de la Estación Experimental IVITA- Huaral. Cuatro cuyes fueron sacrificados a la recepción en granja para la evaluación bacteriológica con el objetivo de comprobar su estado libre de Salmonella.

Se emplearon 10 pozas de material noble de $1.20 \mathrm{~m}$ de largo, $1.0 \mathrm{~m}$ de ancho y 0.56 $\mathrm{m}$ de altura, que fueron divididas a su vez en cuatro pozas individuales de $0.30 \mathrm{~m}^{2}$ de área para cada unidad experimental. Se utilizó viruta de madera como material de cama y se le dispuso de un comedero y un bebedero. Los pesos individuales de cada tratamiento y el peso del alimento se tomaron con una balanza de precisión de $\pm 0.5 \mathrm{~g}$.

Todos los grupos recibieron una alimentación diaria compuesta por $200 \mathrm{~g}$ de alfalfa fresca de la variedad Moapa, alimento terminado en cantidad equivalente al $6 \%$ de su peso vivo en materia seca y agua fresca ad libitum.
La alimentación fue suministrada desde el destete hasta culminar la fase experimental del trabajo. El concentrado fue ofrecido diariamente a las 08:30 y el forraje al medio día, previamente pesados. La formulación del alimento concentrado se realizó en el Laboratorio de Bioquímica, Nutrición y Alimentación de la FMV-UNMSM y estuvo compuesto de afrecho $(52.6 \%)$, maíz grano molido $(22.1 \%)$, torta de soya (13.1\%), soya integral $(5.8 \%)$, melaza (4.1\%), carbonato de calcio $(2.1 \%)$ y sal $(0.3 \%)$.

La distribución de los cuyes fue al azar en cuatro tratamientos con 10 repeticiones cada uno: T1, solución salina; T2, solución salina + APC; T3, desafío experimental con Salmonella Typhimurium; T4, desafío experimental con Salmonella Typhimurium + APC. El antibiótico promotor de crecimiento (APC) utilizado fue zinc bacitracina al 10\%, adquirido de una empresa comercial registrada de productos de uso veterinario, a una dosis de $5 \mathrm{~g}$ por cada 100 kilos de alimento terminado (Navarro, 2007). En el día 11, los animales del $\mathrm{T} 1 \mathrm{y}$ del $\mathrm{T} 2$ fueron dosificados vía oral con $0.5 \mathrm{ml}$ de una solución salina fisiológica, mientras que los del grupo T3 y T4 fueron desafiados con Salmonella Typhimurium a una dosis infectiva de $2 \times 10^{6}$ UFC, vía oral, por única vez. El cultivo de la bacteria y preparación del inóculo fue realizado en el Laboratorio de Microbiología y Parasitología Veterinaria de la FMVUNMSM.

El periodo experimental fue de ocho semanas. Los parámetros productivos evaluados fueron ganancia de peso, consumo de alimento, índice de conversión alimenticia, rendimiento de la canal, retribución económica en base a la alimentación y calidad microbiológica. Al término de la fase experimental se procedió al faenamiento de los animales y se colectaron muestras de pulmón, bazo, vesícula biliar, hígado y ganglios linfáticos mesentéricos además de muestras de las carcasas: pierna, costillar y brazo para el análisis microbiológico. 
Cuadro 1. Parámetros productivos posdestete (2-8 semanas de edad) de cuyes con y sin desafío con Salmonella Typhimurium y con o sin tratamiento con zinc bacitracina (10 cuyes por tratamiento)

\begin{tabular}{lcccc}
\hline \multirow{2}{*}{ Parámetros } & \multicolumn{4}{c}{ Tratamientos } \\
\cline { 2 - 5 } & $\mathrm{T} 1$ & $\mathrm{~T} 2$ & $\mathrm{~T} 3$ & $\mathrm{~T} 4$ \\
\hline Peso inicial (g) & 240 & 248 & 247 & 230 \\
Peso final (g) & $1011^{\mathrm{b}}$ & $1076^{\mathrm{c}}$ & $781^{\mathrm{a}}$ & $807^{\mathrm{a}}$ \\
Ganancia de peso total (g) & $761^{\mathrm{b}}$ & $828^{\mathrm{c}}$ & $534^{\mathrm{a}}$ & $577^{\mathrm{a}}$ \\
Consumo total de alimento (g MS) & $3066^{\mathrm{a}}$ & $3019^{\mathrm{a}}$ & $3327^{\mathrm{b}}$ & $3385^{\mathrm{b}}$ \\
Conversión alimenticia & $4.04^{\mathrm{a}}$ & $3.66^{\mathrm{a}}$ & $6.29^{\mathrm{b}}$ & $5.92^{\mathrm{b}}$ \\
Peso de la canal (g) & $713^{\mathrm{b}}$ & $760^{\mathrm{c}}$ & $554^{\mathrm{a}}$ & $574^{\mathrm{a}}$ \\
Rendimiento de canal (\%) & $71.3^{\mathrm{a}}$ & $70.6^{\mathrm{a}}$ & $70.9^{\mathrm{a}}$ & $71.2^{\mathrm{a}}$ \\
\hline
\end{tabular}

Promedios dentro de filas con letras diferentes son estadísticamente diferentes $(p<0.05)$

T1, solución salina; T2, solución salina + APC; T3, desafío con Salmonella Typhimurium; T4, desafío con Salmonella Typhimurium + APC

Los datos se analizaron con el paquete estadístico SAS ${ }^{\circledR}$ Estudio (Statistical Analisis System) con un nivel de significación de 0.05 , utilizando el análisis de varianza y la prueba de Duncan para determinar diferencias estadísticas entre tratamientos.

\section{Resultados}

\section{Ganancia de peso}

El mayor peso final se presentó en los animales del T2 (Cuadro 1). Además, se encontró diferencia significativa $(\mathrm{p}<0.05)$ entre los tratamientos de los animales desafiados a Salmonella (T3 y T4) frente a los animales de T1 y T2. No hubo diferencia significativa en peso final entre los animales desafiados con Salmonella (T3 y T4).

Las diferencias en ganancia de peso y ganancia de peso total fueron significativas a partir de la tercera semana entre los grupos T1 y T2 comparado con los grupos T3 y T4 $(\mathrm{p}<0.05$ ). Los cuyes de T2 presentaron $20.2 \%$ más de ganancia de peso frente al grupo con- trol (T1), pero esta diferencia no fue estadísticamente significativa.

\section{Consumo de Alimento}

Los cuyes de los tratamientos T3 y T4 presentaron $10 \%$ de consumo adicional en comparación con los cuyes de T1 y T2 (Cuadro $1 ; \mathrm{p}<0.05)$. Estas diferencias solo fueron evidentes entre las semanas 2 y 5 , toda vez que entre las semanas 6 y 8 la diferencia del consumo de alimento fue similar entre tratamientos. Los cuyes del T2 presentaron el menor consumo de alimento (3019 g).

\section{Índice de Conversión Alimenticia}

Los cuyes de T1 y T2 mostraron una mejor conversión alimenticia frente a los cuyes de T3 y T4 (Cuadro 1). La conversión alimenticia de T2 fue $10.38 \%$ mejor que en T1, aunque esta diferencia no fue estadísticamente significativa. En forma similar entre los grupos de cuyes desafiados con Salmonella (T3 y T4), los cuyes de T4 mostraron un 5.9\% mejor conversión alimenticia que los de $\mathrm{T} 3$, pero sin diferencia significativa. 
Cuadro 2. Conversión alimenticia semanal de cuyes con y sin desafío con Salmonella Typhimurium y con o sin tratamiento con zinc bacitracina (10 cuyes por tratamiento)

\begin{tabular}{lcccc}
\hline \multirow{2}{*}{ Semana } & \multicolumn{4}{c}{ Tratamientos } \\
\cline { 2 - 5 } & $\mathrm{T} 1$ & $\mathrm{~T} 2$ & $\mathrm{~T} 3$ & $\mathrm{~T} 4$ \\
\hline Primera & $2.66^{\mathrm{b}}$ & $2.01^{\mathrm{a}}$ & $2.34^{\mathrm{ab}}$ & $2.60^{\mathrm{b}}$ \\
Segunda & $2.83^{\mathrm{ab}}$ & $2.59^{\mathrm{a}}$ & $3.34^{\mathrm{c}}$ & $3.21^{\mathrm{bc}}$ \\
Tercera & $4.26^{\mathrm{a}}$ & $3.39^{\mathrm{a}}$ & $9.22^{\mathrm{b}}$ & $4.59^{\mathrm{a}}$ \\
Cuarta & $3.92^{\mathrm{a}}$ & $3.58^{\mathrm{a}}$ & $10.12^{\mathrm{b}}$ & $10.22^{\mathrm{b}}$ \\
Quinta & $4.51^{\mathrm{a}}$ & $4.65^{\mathrm{a}}$ & $10.76^{\mathrm{b}}$ & $11.17^{\mathrm{b}}$ \\
Sexta & $5.09^{\mathrm{a}}$ & $4.63^{\mathrm{a}}$ & $8.30^{\mathrm{b}}$ & $8.01^{\mathrm{b}}$ \\
Séptima & $4.96^{\mathrm{a}}$ & $4.43^{\mathrm{a}}$ & $7.08^{\mathrm{b}}$ & $7.27^{\mathrm{b}}$ \\
Octava & $4.54^{\mathrm{a}}$ & $4.24^{\mathrm{a}}$ & $6.88^{\mathrm{b}}$ & $5.16^{\mathrm{a}}$ \\
\hline Total & $4.04^{\mathrm{a}}$ & $3.66^{\mathrm{a}}$ & $6.29^{\mathrm{b}}$ & $5.92^{\mathrm{b}}$ \\
\hline
\end{tabular}

Promedios dentro de filas con letras diferentes son estadísticamente diferentes $(p<0.05)$

T1, solución salina; T2, solución salina + APC; T3, desafío con Salmonella Typhimurium; T4, desafío con Salmonella Typhimurium + APC

En la tercera semana se observa diferencias significativas entre $\mathrm{T} 1, \mathrm{~T} 2$ y $\mathrm{T} 4$ frente a $\mathrm{T} 3$, mientras que a partir de la cuarta semana se hacen evidentes las diferencias de los grupos no desafiados (T1 y T2) frente a los grupos desafiados (T3 y T4) $(\mathrm{p}<0.05$; Cuadro 2).

\section{Rendimiento de la Canal}

Las canales de los cuyes incluyen la cabeza, patas, hígado, corazón, pulmones y riñones. El peso de la canal fue mayor en T2, seguido de T1, y fue menor en los grupos de animales desafiados con Salmonella (T3 y T4), no habiendo diferencias significativas entre estos últimos (Cuadro 1). No obstante, el rendimiento de la canal expresado en porcentaje fue similar entre los cuatro tratamientos.

\section{Retribución Económica}

La retribución económica se presenta en el Cuadro 3. Para este cálculo se consideró el precio de venta de S/. 23.00 por kilo de carne de cuy, precio del cuy ofertado por la Estación Experimental IVITA Huaral de la FMV-UNMSM. Los cuyes alimentados con APC y no desafiados con Salmonella (T2) mostraron una mejor retribución económica, seguido por el grupo control (T1), mientras que los animales desafiados con Salmonella Typhimurium reportan una pérdida del 28 al $30 \%$.

\section{Calidad Microbiológica}

No se presentaron casos de mortalidad durante las ocho semanas del estudio; sin embargo, durante las necropsias realizas al beneficio de los animales se detectaron focos necróticos en el hígado y bazo, así como bazo agrandado y pulmones congestionados en tres animales de T3 y dos de T4 (Figuras 1 y 2 ).

La presencia de Salmonella en los órganos y canal de los cuyes se muestra en el Cuadro 4. En T1 (grupo control) se presentó en los pulmones de dos cuyes y en el bazo de 
Cuadro 3. Retribución económica en base a la alimentación de cuyes con y sin desafío con Salmonella Typhimurium y con o sin tratamiento con zinc bacitracina (10 cuyes por tratamiento)

\begin{tabular}{lcccc}
\hline & $\mathrm{T} 1$ & $\mathrm{~T} 2$ & $\mathrm{~T} 3$ & $\mathrm{~T} 4$ \\
\hline Peso de la canal (g) & 713 & 760 & 554 & 574 \\
Precio cuy/kg (S/) & 23 & 23 & 23 & 23 \\
Ingreso bruto/canal (S/) & 16.40 & 17.48 & 12.74 & 13.20 \\
Alimento balanceado & & & & \\
$\quad$ Consumo (g) & 983.09 & 941.63 & 1218.16 & 1269.13 \\
$\quad$ Costo/kg (S/) & 1.70 & 1.70 & 1.70 & 1.70 \\
$\quad$ Subtotal (S/) & 1.67 & 1.60 & 2.07 & 2.16 \\
Forraje alfalfa & & & & \\
$\quad$ Consumo (g) & 11,200 & 11,200 & 11,200 & 11,200 \\
$\quad$ Costo/kg (S/) & 0.10 & 0.10 & 0.10 & 0.10 \\
$\quad$ Subtotal (S/) & 1.12 & 1.12 & 1.12 & 1.12 \\
Costos de alimentación & & & & \\
$\quad$ APC (zinc bacitracina) (S/) & - & 0.10 & - & 0.10 \\
$\quad$ Costo de alimentación (S/) & 2.79 & 2.82 & 3.19 & 3.38 \\
$\quad$ Retribución económica (S/) & 13.61 & 14.66 & 9.55 & 9.82 \\
$\quad \begin{array}{l}\text { Retribución económica } \\
\text { relativa }\end{array}$ & 100.0 & 107.7 & 70.2 & 72.2 \\
\hline
\end{tabular}

T1, solución salina; T2, solución salina + APC; T3, desafío con Salmonella Typhimurium; T4, desafío con Salmonella Typhimurium + APC
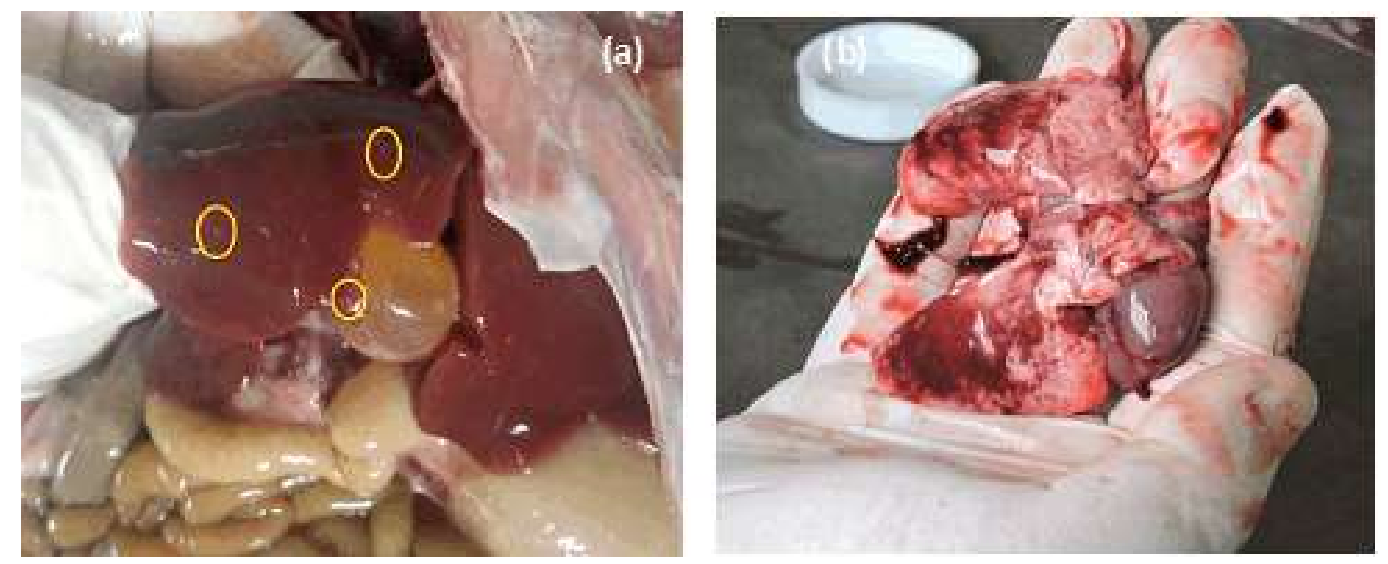

Figura 1. Hallazgos en la necropsia de cuyes desafiados con Salmonella Typhimurium (a) Presencia de focos necróticos en hígado; (b) Congestión a nivel pulmonar 
Cuadro 4. Número de casos con presencia de Salmonella en los órganos y canal de cuyes con y sin desafío con Salmonella Typhimurium y con o sin tratamiento con zinc bacitracina (10 cuyes por tratamiento)

\begin{tabular}{ccccccc}
\hline Tratamiento & Pulmón & Bazo & $\begin{array}{c}\text { Vesícula } \\
\text { biliar }\end{array}$ & Hígado & $\begin{array}{c}\text { Ganglios } \\
\text { linfáticos } \\
\text { mesentéricos }\end{array}$ & Canal \\
\hline T1 & 2 & 1 & - & - & - & - \\
T2 & - & - & - & - & - & - \\
T3 & 7 & 9 & 7 & 9 & 10 & 4 \\
T4 & 6 & 5 & 4 & 6 & 7 & - \\
\hline
\end{tabular}

T1, solución salina; T2, solución salina + APC; T3, desafío con Salmonella Typhimurium; T4, desafío con Salmonella Typhimurium + APC

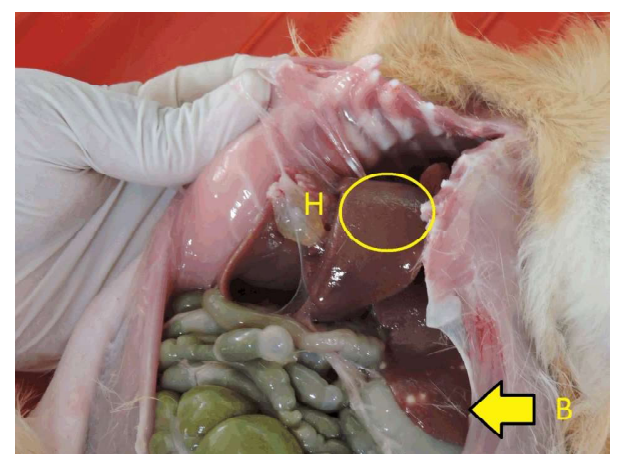

Figura 2. Focos necróticos en el hígado $(\mathrm{H})$ y esplenomegalia y presencia de focos necróticos en el bazo de un cuy desafiado con Salmonella Typhimurium

un cuy, pero en ningún caso en los cuyes de T2 (con APC). En general, Salmonella fue más frecuentemente aislada de los órganos y canal de cuyes de $\mathrm{T} 3$ en relación con los cuyes de T4 tratados con APC.

\section{Discusión}

La ganancia de peso de los cuyes control (T1) y los tratados con APC zinc bacitracina (T2) fue superior a los $693 \mathrm{~g}$ reportado por Sánchez et al. (2013) por un periodo de 67 días y a los 620-668 g reportados por Mamani et al. (2015) en un periodo de
12 semanas, en ambos casos en cuyes al pastoreo en la Estación Experimental del IVITAMantaro en Huancayo, a pesar de ser tener una calidad genética similar.

La ganancia de peso de los cuyes en T1 y T2 fue similar. En este sentido, Torres et al. (2013) tampoco encontraron diferencias significativas entre cuyes control y tratados con zinc bacitracina en una dosis de $0.1 \%$ por kg de afrecho (616 y $660 \mathrm{~g}$, respectivamente) durante 55 días. En forma similar, Valdizán (2018) dosificó cuyes con $300 \mathrm{~g}$ de zinc bacitracina por tonelada de afrechillo (697 g) sin encontrar diferencias estadísticas frente al grupo control (684 g), mientras que Gonzáles (2009), en trabajos realizados con pavos de engorde y suplementados con zinc bacitracina $(0.035 \%)$ tampoco encontraron diferencias significativas con el grupo control.

El consumo de alimento fue mayor en los animales desafiados con Salmonella (T3: 3,327 y T4: 3,385 g MS, respectivamente), resultado similar a lo reportado por Torres et al. (2013) al tratar cuyes con una cepa probiótica. No obstante, al comparar estos consumos con otros estudios se puede indicar que el consumo de alimento estuvo dentro del rango promedio (Chauca, 1997). El $7.22 \%$ de mayor consumo de alimento de los grupos desafiados en comparación con los 
cuyes que no fueron desafiados (T1 y T2) es contradictorio con los resultados en otros estudios. Así, Torres et al. (2013) obtuvieron un menor consumo en el grupo que recibió zinc bacitracina frente al grupo control, mientras que Valdizán (2018) obtuvo un consumo de MS de $2773 \mathrm{~g}$ en los cuyes tratados con zinc bacitracina frente a los animales control (3083 g) en 56 días.

Los índices de conversión alimenticia en los grupos desafiados con Salmonella fueron similares a los reportados por Torres et al., (2013), quienes obtuvieron una conversión alimenticia entre 3.90 y 5.04 al utilizar zinc bacitracina en cuyes. Los índices de conversión alimenticia en T3 y T4 fueron elevados (6.29 y 5.92, respectivamente), aunque similares a los reportados por Castillo et al. (2012) en cuyes suplementados con bloques minerales (6.9 en su grupo control) y por Choez et al. (2018), con índices de 6.03-6.91 en cuyes alimentados con frejol castilla.

En estudios con otras especies, no obstante, se obtuvieron mejores resultados con el uso del zinc bacitracina. Así, Gonzáles (2009) no encontró diferencias significativas entre pavos de engorde dosificados con zinc bacitracina (1.98 \pm 0.17$)$ y el grupo control (2.05 \pm 0.17$)$, al igual que Quispe (2014) con pollos desafiados con Eimeria spp y Clostridium perfringens dosificados con zinc bacitracina, debido a la acción del APC que altera la composición y la actividad de la microflora.

El rendimiento de la canal fue similar a los resultados reportados por Torres et al. (2013) de $71.7 \pm 2.6 \%$ en cuyes dosificados con zinc bacitracina y a los de Yamada et al. (2019) de 72.7 y $72.3 \%$ en una comparación de dos líneas cárnicas de cuyes en la costa. Aybar (2011), asimismo, reportó $67.8 \%$ de rendimiento de canal en cuyes sometidos a una alimentación a base de alfalfa verde y de $71.0 \%$ para cuyes que recibieron una alimentación mixta, mientras que Yoplac et al. (2017) reportó rendimientos de canal entre $70.8 \mathrm{y}$ $71.5 \%$ en cuyes alimentados con pulpa de café. Resultados menores sobre rendimiento de canal fueron reportados por Castilla (2018) con $65.0-66.6 \%$ en cuyes alimentados con frejol castilla durante 7 semanas y por Chauca (1997) con $67.4 \%$ de rendimiento de canal en cuyes mejorados criados en pozas.

Según los resultados obtenidos, la Salmonella afecta seriamente el rendimiento productivo de los animales desafiados experimentalmente causando una menor retribución económica en la venta de carne, llegando a mostrar pérdidas entre el 28 y $30 \%$. En el análisis microbiológico, los hallazgos en animales desafiados con Salmonella (T3) fueron similares a lo reportado por Matsuura et al. (2010) en hígados de cuyes clínicamente enfermos.

\section{Conclusiones}

- Los parámetros productivos y la retribución económica en cuyes se afectan negativamente por la infección subclínica con Salmonella Typhimurium.

- El uso en la dieta de zinc bacitracina a dosis de 50 ppm en animales desafiados con Salmonella Typhimurium disminuyó los efectos negativos producidos por el patógeno

\section{Litigratura Citada}

1. Aybar M. 2011. Perfil lipídico sanguíneo de cuyes en crecimiento en el C.E. Pampa del Arco - Ayacucho. Tesis de Médico Veterinario. Ayacucho, Perú: Univ. Nacional San Cristóbal de Huamanga. 98 p.

2. Borrelli L, Fioretti A, Ruggiero V, Santaniello A, Grincoli G, Ricci A, Barco L, et al. 2011. Salmonella Typhimurium DT104 in farmed rabbits. J Vet Med Sci 73: 385-387. doi: 10.1292/ jvms.10-0315

3. Bustamante J. 1993. Producción de cuyes. Lima: Univ. Nacional Mayor de San Marcos. 259 p. 
4. Carroll K. 2014. Bacteriología. En: Microbiología médica. 26a ed. México: McGraw Hill. p 229-243.

5. Castillo C. Carcelén F. Quevedo W. Ara M. 2012. Efecto de la suplementación con bloques minerales sobre la productividad de cuyes alimentados con forraje. Rev Inv Vet Perú 23: 414-419. doi: 10.15381/rivep.v23i4.946

6. Chauca L. 1994. Fisiología digestiva de los cuyes. Lima, Perú: Instituto Nacional de Investigaciones Agraria INIA. 11p.

7. Chauca L. 1997. Producción de cuyes (Cavia porcellus). Roma: Organización de las Naciones Unidas para la Agricultura y la Alimentación - FAO. 77 p.

8. Chauca L, Peruano D, Muscari J. 1997. Comportamiento reproductivo de gestación postpartum y postdestete de cuyes (Cavia porcellus) manejados en empadre continuo durante un año. En: XX Reunión de la Asociación Peruana de Producción Animal. Tingo María. Perú.

9. Chero A. 2015. Identificación molecular de Salmonella Typhimurium y entiritidis en cobayos reproductoras primerizas clínicamente sanas. Tesis de Médico Veterinario. Lima: Univ. Nacional Mayor de San Marcos. $54 \mathrm{p}$.

10. Choez, K, Ravillet V. 2018. Frejol castilla (Vigna unguiculata 1. Walp) como ingrediente en raciones de crecimiento-engorde de cuyes (Cavia porcellus) mejorados. Rev Inv Vet Perú 29: 180-187. doi: 10.15381/rivep.v29i1.14086

11. Evans $A$. 2005. Import risk analysis: domestic guinea pig. Cavia porcellus imported from Australia [Internet]. Disponible en: http:/hintlink.com/guinea_pig/Nzriskanalysis.pdf

12. Gonzáles H. 2009. Evaluación de la harina de yacón (Smallanthus sonchifolius) como prebióticos en la dieta de pavos de engorde. Tesis de Médico Veterinario. Lima: Univ. Nacional Mayor de San Marcos. 94 p.
13. Hirikawa H. 2001. Coprophagy in leporids and other mammalian herbivores. Mammal Rev 31: 61-80. doi: 10.1046/ j.1365-2907.2001.00079.x

14. Layme A. 2010. Frecuencia de lesiones anatomopatológicas en cobayos con diagnóstico bacteriológico de Salmonella sp remitidos al Laboratorio de Histología, Embriología y Patología Veterinaria de la FMV-UNMSM durante el periodo 2001-2007. Tesis de Médico Veterinario. Lima, Perú: Univ. Nacional Mayor de San Marcos. 64 p.

15. Matsuura A, Morales $S$, Calle $S$, Ara M. 2010. Susceptibilidad a antibacterianos in vitro de Salmonella enterica aislada de cuyes de crianza familiar-comercial en la Provincia de Carhuaz, Ancash. Rev Inv Vet Perú 21: 93-99. doi: 10.15381/rivep.v21i1.355

16. Merck \& Co. 2007. Manual Merck de veterinaria. $9^{\circ}$ ed. Barcelona, España: Ed Océano. 2063 p.

17. Molina M. 2008. Efecto probiótico de Lactobacillus acidophilus y Bacillus subtilis en cuyes (Cavia porcellus) de engorde. Tesis de Ingeniero Agropecuario. Sangolqui, Ecuador: Escuela Politécnica del Ejército. $118 \mathrm{p}$.

18. Morales S, Mattos J, Calle S. 2007. Efecto de la muña (Satureja parvifolia) en la dinámica de la infección por Salmonella enterica en cobayos. En: XXX Reunión Científica Anual Asociación Peruana de Producción Animal. Cusco, Perú.

19. Navarro A. 2007. Antibióticos de uso veterinario y su relación con la seguridad alimentaria y salud pública. Madrid, España. [Internet]. Disponible en: http:// www.racve.es/files/2013/03/2007-0210 -Discurso-ingreso-D.-ArturoRam\%C3\%B3n-Anad\%C3\%B3nNavarro.pdf

20. Ordoñez R. 1998. Efecto de dos niveles de proteína y fibra cruda en el alimento de cuyes (Cavia porcellus) en lactación y crecimiento. Tesis de Ingeniero Zootecnista. Lima, Peerú: Univ. Nacional Agraria La Molina. 65 p. 
21. Quispe V. 2014. Efecto de tres promotores de crecimiento sobre los parámetros productivos en pollos de engorde desafiados experimentalmente con Clostridium perfringens. Tesis de Médico Veterinario. Lima, Perú: Univ. Nacional Mayor de San Marcos. 66 p.

22. Sánchez R, Jiménez $R$, Huamán $H$, Bustamante J, Huamán A. 2013. Respuesta productiva y económica al uso de cuatro tipos de comederos para forraje en la crianza de cuyes. Rev Inv Vet Perú 24: 441-450. doi: 10.15381/rivep.v24i4. 2733

23. Torres C, Carcelén F, Ara M, San Martín F, Jiménez R, Quevedo W, Rodríguez J. 2013. Efecto de la suplementación de una cepa probiótica sobre los parámetros productivos del cuy
(Cavia porcellus). Rev Inv Vet Perú 24: 433-440. doi: 10.15381/rivep.v24i4.2729

24. Valdizán C. 2018. Efecto de la inclusión de probiótico, prebiótico y simbiótico en la dieta del cuy (Cavia porcellus) sobre parámetros productivos. Tesis de Médico Veterinario. Lima, Perú: Univ. Nacional Mayor de San Marcos. 66 p.

25. Yamada G Bazán V, Fuentes N. 2019. Comparación de parámetros productivos de dos líneas cárnicas de cuyes en la costa central del Perú. Rev Inv Vet Perú 30: 240-246. doi: 10.15381/rivep.v$30 \mathrm{i} 1.15678$

26. Yoplac I, Yalta J, Vásquez H, Maicelo J. 2017. Efecto de la alimentación con pulpa de café (Coffea arabica) en los índices productivos de cuyes (Cavia porcellus L.) raza Perú. Rev Inv Vet Perú 28: 549-561. 10.15381/rivep.v-28i3.13362 